\title{
Applications of CMOS visible image sensor to survey of potentially hazardous asteroids using optimized ground based telescopes
}

Stephen M. Larson, Gary R. Sims, Eric J. Christensen

Stephen M. Larson, Gary R. Sims, Eric J. Christensen, "Applications of CMOS visible image sensor to survey of potentially hazardous asteroids using optimized ground based telescopes," Proc. SPIE 10706, Advances in Optical and Mechanical Technologies for Telescopes and Instrumentation III, 107063Q (10 July 2018); doi: 10.1117/12.2314081

Event: SPIE Astronomical Telescopes + Instrumentation, 2018, Austin, Texas, United States 


\title{
Application of CMOS visible Image sensor to survey of potentially hazardous asteroids using optimized ground based telescopes Stephen M. Larson ${ }^{\mathrm{a}}$, Gary R. Sims ${ }^{\mathrm{b}}$, Eric J. Christensen ${ }^{\mathrm{a}}$ \\ ${ }^{a}$ Lunar and Planetary Laboratory, 1629 E. University Blvd., University of Arizona, Tucson, AZ 85721 , 'Spectral Instruments, Inc., 420 N. Bonita Avenue, Tucson, AZ 85745
}

\begin{abstract}
Application of CMOS image sensors with non-destructive readout capability have several advantages over current CCD sensors in detecting Near-Earth Objects (NEOs). They include detection of temporal changes, cosmic ray rejection, no charge blooming, expanded dynamic range, and lower dark current. Since wide field survey usually requires large mosaics, a "rolling shutter" operation simplifies the challenge of large mechanical shutters. Being able to readout parts of the field in destructive mode offers the possibility of providing guiding feedback to the telescope during exposure. We have carried out preliminary testing of a prototype CMOS camera built by Spectral Instruments Inc. on a one-meter telescope on Mt. Lemmon, Arizona as applied to rapidly moving NEOs. We have also demonstrated "post facto" guiding on a known NEO that significantly improves the signal to noise.
\end{abstract}

Keywords: CMOS image sensor, ground-based astronomy, non-destructive readout

\section{INTRODUCTION}

NASA's Planetary Defense Program is tasked with detecting and tracking potentially hazardous near-Earth objects (NEOs) as small as 40 meters in diameter that might reach Earth's surface. Currently, there are three major survey telescopes accounting for $93 \%$ of the 690 discoveries so far in 2018 .

Surveying for Near-Earth Objects (NEOs) differs from other types of surveys because NEOs are moving relative to the background star field and subject to trailing losses during an exposure. This effect can be minimized with sensor arrays with high DQE, coupled to a telescope with a fast optical speed - the same requirements for artificial satellite detection needed for space situational awareness.

For example, the Catalina Sky Survey for NEOs on Mt. Lemmon in Arizona utilizes a 1.5-m, f/1.6 reflector with a thinned, backside illuminated STA $160010.5 \mathrm{k} \mathrm{x} 10.5 \mathrm{k} \mathrm{CCD}$ at the prime focus. Current survey telescopes employ CCDs either with a monolithic 111 megapixel device (Catalina Sky Survey), or mosaic of 60 OTC CCDs with 1.4 gigapixels (PanSTARRS in Hawaii). The Catalina Sky Survey also operates a 1-m, f/2.6 follow-up telescope located near the main survey telescope.

The surveys typically use cadences of 4 images of a field separated by 25-20 minutes and employs software that identifies objects that move in linear fashion relative to the background star field. Candidate asteroids are validated visually before their astrometric positions are reported to the Minor Planet Center, who determine that the object is not known and posting a short-arc orbit ephemeris for observers (mostly amateurs) can obtain follow-up positions to extend the arc and reduce the orbit uncertainty.

NEOs that are relatively close to the Earth are often streaked over the 30-60 exposures, and are subject to trailing losses resulting in reduced signal to noise ratio. Once the rate and direction of apparent motion is known, it is possible to take a series of shorter exposures and register them using a "stack and add" method to overcome the trailing loss. 


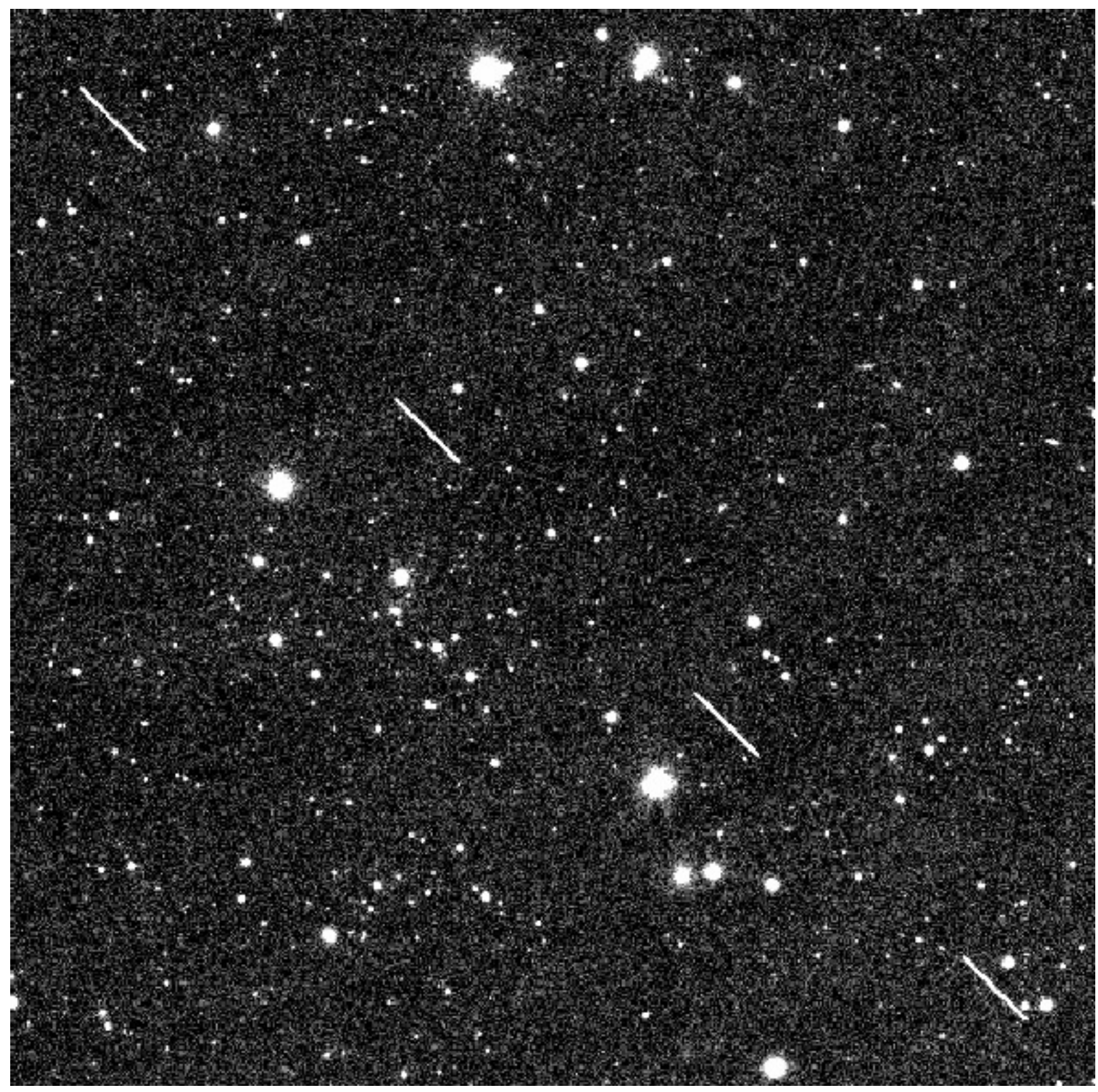

Figure 1. The fast moving $25-\mathrm{m}$ diameter NEO $2008 \mathrm{FP}$ that came within 0.4 lunar distance moving at $33 \mathrm{~km} / \mathrm{sec}$. This is a composite of four 5 second exposures. The gaps between streaks is due to the CCD $13 \mathrm{sec}$ readout time. 


\section{APPLYING CMOS SENSORS FOR NEO DETECTION}

Current CMOS devices have several advantages over the traditional CCD in astronomical applications. They include:

- No need for mechanical shutter ("rolling" shutter"). This is helpful for mosaic arrays used in large aperture, large field focal planes.

- No blooming from saturated pixels which destroys data in the vicinity of a bright object.

- Lower dark current. As low as 7e-/pixel/hour measured @ -70C.

- Lower effective readout noise. Measured 6 electrons/frame on a 2K x 2K sensor @ 5frames per second.

- Increased dynamic range due to a high pixel well capacity.

- Cosmic ray rejection by locating and eliminating discontinuities in each pixel signal ramp.

- Possibility of on-chip astronomical guiding by assigning regions of destructive, and non-destructive pixels for data acquisition and temporal guiding off background stars.

- Acquiring temporal data on objects of varying brightness, such as rotational light curves of elongated asteroids.

- DQE of frontside illuminated destructive readout CMOS is typically $65 \%$ vs. $45 \%$ for CCDs. Thinned, back illuminated CMOS are similar to back illuminated CCDs.

Details of Spectral Instrument's prototype CMOS camera can be found in Sims, et al. elsewhere in this meeting.

Proc. of SPIE Vol. 10706 107063Q-3 


\section{ON-SKY EXPERIMENT}

We have mounted the Spectral Instruments prototype CMOS camera to the Steward Observatory 1-m follow-up telescope at the Mt. Lemmon Observatory (9157 ft elevation) just north of Tucson, Arizona. This telescope was upgraded specifically for rapid follow-up of candidate NEOs that requires only a half degree usable field for a $2 \mathrm{k} x$ $2 \mathrm{~K}$ sensor with 15 micron pixels.

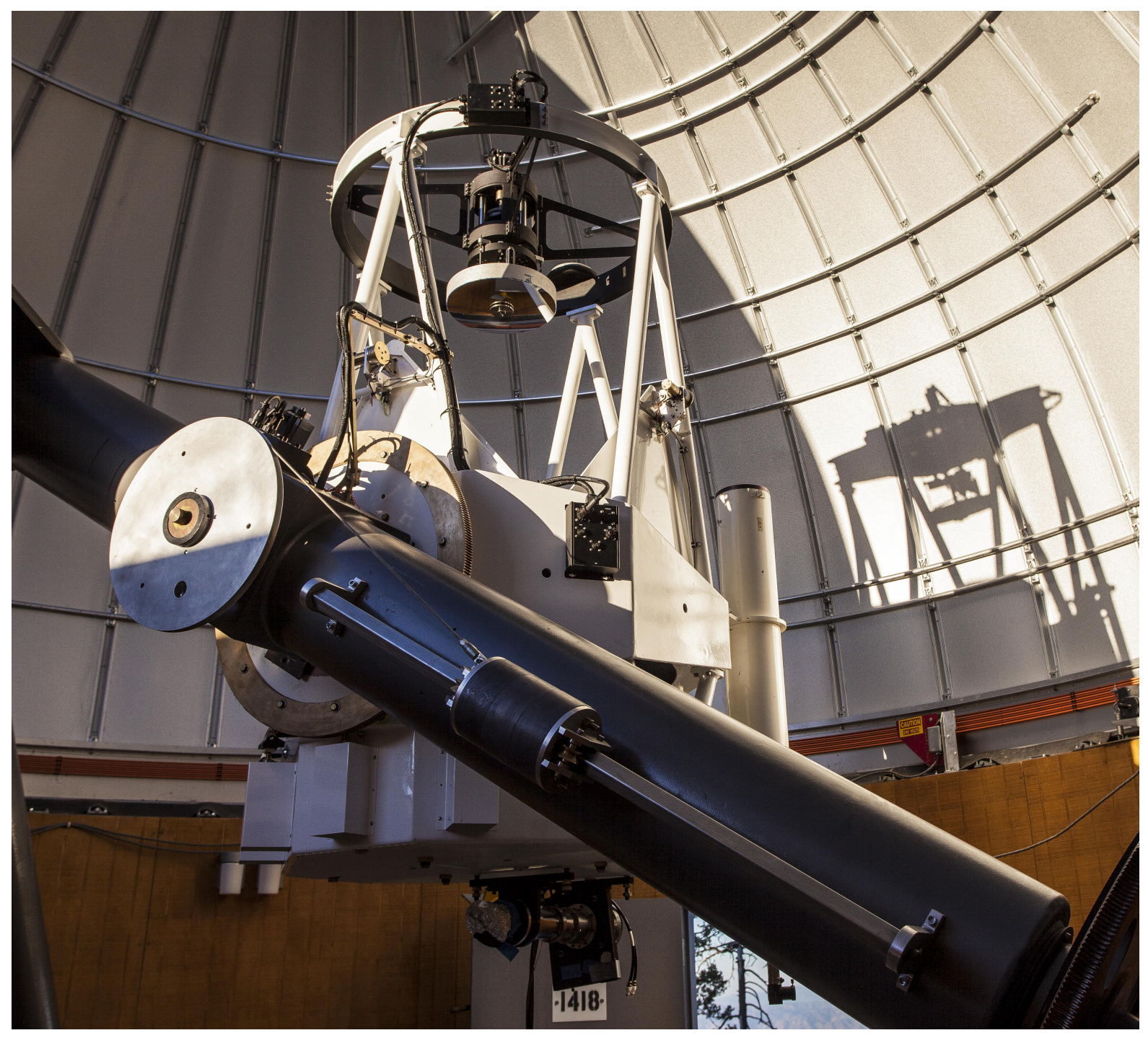

Figure 2. The 1-m follow-up telescope on Mt. Lemmon used for this study. The usable focal plane is 0.5 degrees across with an $\mathrm{f} / 2.6$ beam. 


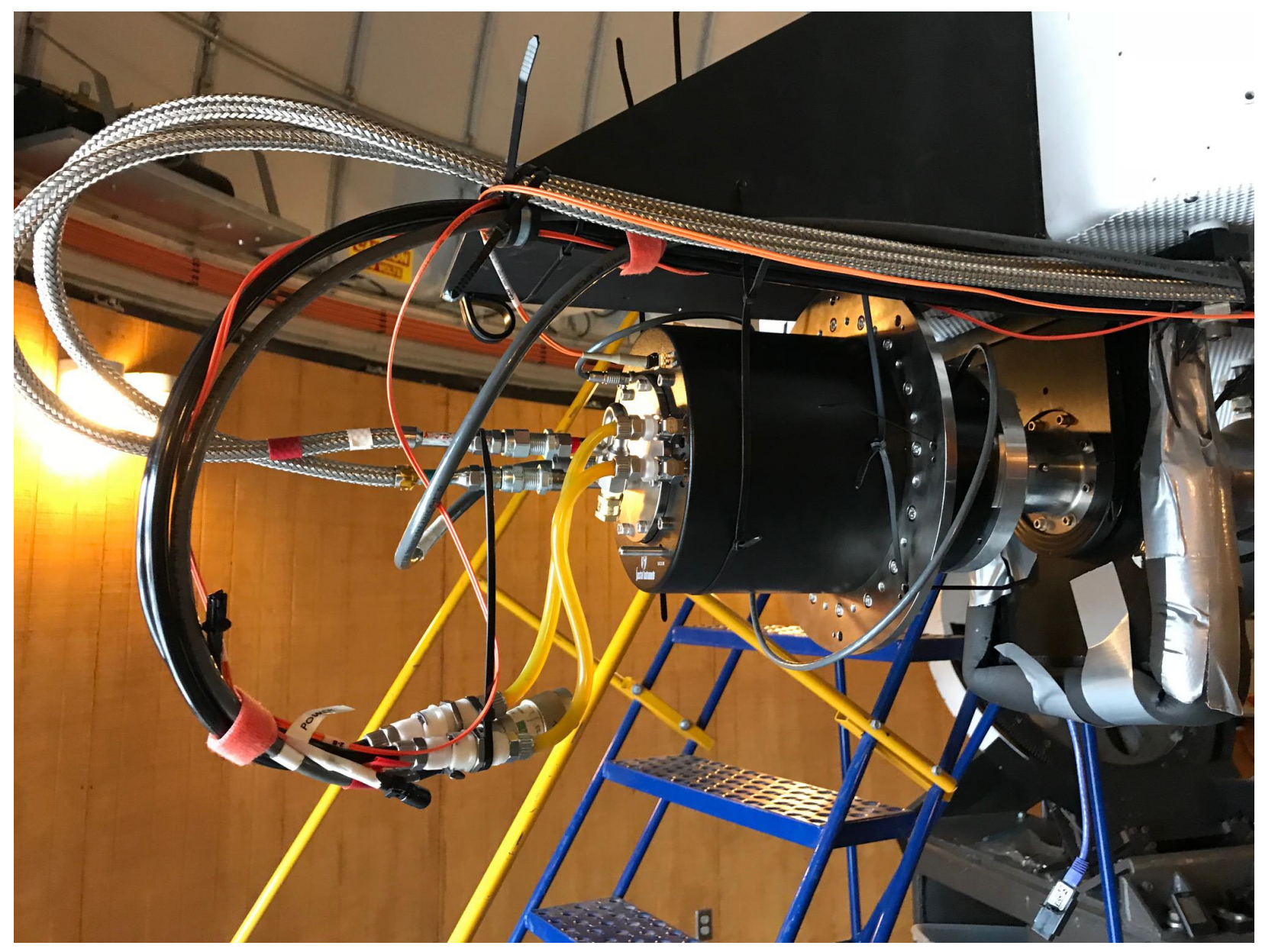

Figure 3. The Spectral Instruments prototype CMOS camera mounted on the 1-m follow-up telescope. The CMOS is cooled to $-100 \mathrm{C}$ by a cryotiger cooler, and the interior electronics are kept cool by circulating cold water. The power line and fiber output can be seen. The A/D converters and associated components are housed in the black canister. 

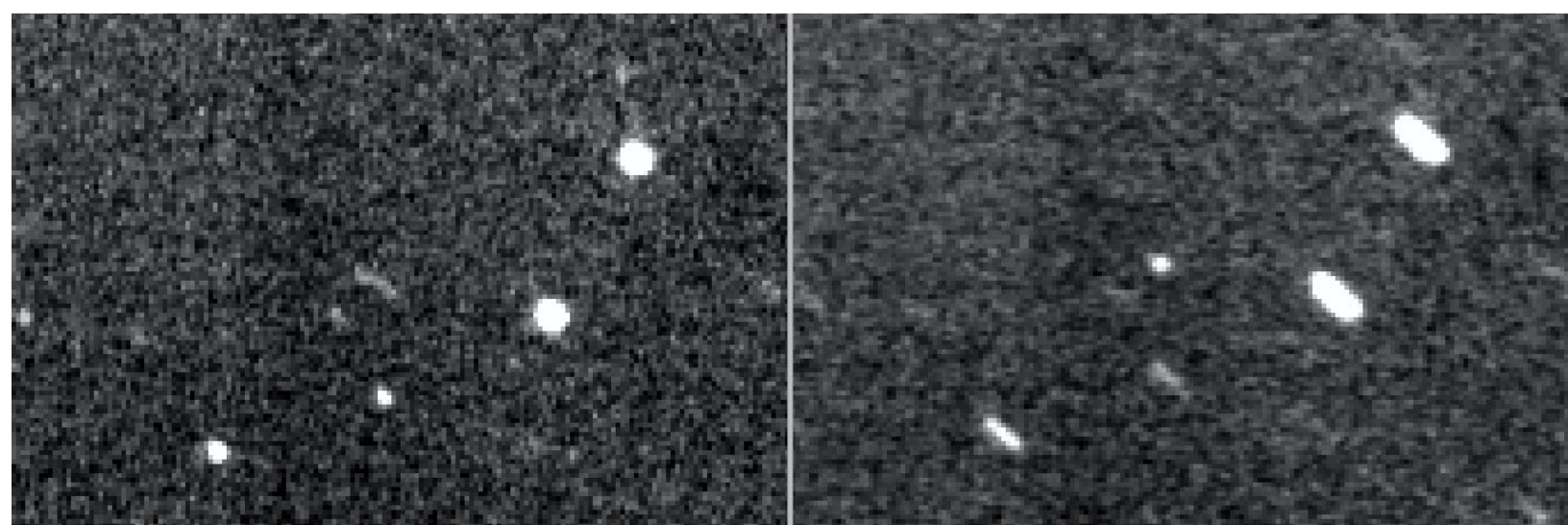

Figure 4. Sample images of a NEO with the Spectral Instruments prototype CMOS camera. The NEO was moving 3.1 degree/day (the short streak in the center of the left panel). The right panel is from 79 "slices" of exposure length 0.3833 seconds each, from 4 exposures separated by only $4 \mathrm{sec}$ gaps registered on the NEO.

\section{FUTURE WORK}

We will be taking more data to demonstrate rotational light curves of asteroids. And better sensitivity relative to our CCDs.

\section{ACKNOWLEDGEMENTS}

Frank Shelly (LPL), Hans Meyer (SI) and William Schempp (SI) contributed to this project. The Catalina Sky Survey is supported by NASA grant NNX09AH26G. 